\title{
Paro Cardiorrespiratorio (PCR) y Reanimación Cardiopulmonar (RCP) en un nuevo escenario: COVID19
}

\section{A new setting:Cardiorespiratory arrest (PCR) and Cardiopulmonary Resuscitation (CPR) in COVID19}

María Mercedes Aguirre C. ${ }^{1}$

\begin{abstract}
Our country, like the rest of the world, have been facing for a little more than three months, a pandemia caused by a new virus called "SARS-CoV-2", which spread started in Wuhan, China. As this virus has crossed all geographic boundaries, so has done with those related to human wellbeing. We have faced social, economic, epidemiologic, organizational, biologic and clinic challenges. The COVID-19, which is the name the World Health Organization (WHO) gave to the disease caused by this virus, uses to show clinical manifestations similar to those caused by a common flu in the vast majority of cases. Although there is a low percentage of patients who developed a severe clinical profile, that makes necessary an Intensive Care Unit (ICU) admission. This virus has the particular feature of being highly transmissible from one person to another by direct contact, droplets and aerosols generation. This has led the number of infected all around the world to grow in an exponential manner, which remains a real hazard for the available resources needed for personal and community protection. The natural questions that arise from this landscape are: what must be done if a COVID+ or suspected, presents a cardiac arrest (CA) and need cardiopulmonary reanimation (CPR)? Should RCP be started considering the patient prognosis and the resources shortage? It seems worthy to take the risk of becoming infected? Am I really at risk of becoming infected if I participate in performing the CPR maneuvers? Do I have to perform the RCP maneuvers following the International Liaison Committee On Reanimation (ILCOR) guides that I already know or there are some differences? And what I am supposed to do if the CA occurs while the patient
\end{abstract}

\section{Key words:}

Cardiac arrest, cardiopulmonar reanimation, COVID-19, reanimation in the prone position

Profesora Asociada. Directora de Departamento de Anestesiología y Medicina Perioperatoria Facultad de Medicina Universidad de Chile.

\section{ORCID}

https://orcid.org/0000-0001-6703-9493

\section{Correspondencia:}

María Mercedes Aguirre C.

Email: mmaguirre@uchile.cl 
is in the prone position? This revision tries to find some of the answers for those questions, based on current publications and published recommendations so far. Certainly, those are scarce in this field and are subject to changes, at least for some months, while the scientific and medical community achieve the consolidated knowledge about the global clinic behavior of this new disease, COVID-19.

\section{RESUMEN}

Nuestro país y el mundo lleva poco más de tres meses enfrentando una pandemia causada por un nuevo virus, el Ilamado SARS-CoV-2, cuya propagación se inició en Wuhan, China. Este virus ha atravesado todas las fronteras geográficas, así como las de aquellas áreas que componen el bienestar del ser humano. Nos hemos visto enfrentados a desafíos sociales, económicos, epidemiológicos, organizacionales, biológicos y clínicos. La enfermedad causada por este virus, llamada por la Organización Mundial de la Salud (OMS) COVID-19, se caracteriza fundamentalmente por presentarse como un cuadro clínico parecido a la influenza en la gran mayoría de los casos, sin embargo, existe un porcentaje de ellos que cursa de manera grave, requiriendo cuidados críticos. Tiene, además, la particularidad de ser altamente transmisible entre las personas a través del contacto directo, generación de gotitas y aerosoles principalmente, lo cual ha llevado a un aumento exponencial del número de contagiados en el mundo, poniendo en jaque la capacidad de contar con el recurso necesario suficiente para la protección personal y de la comunidad. Surge naturalmente la interrogante: ¿Qué hacemos si un paciente COVID-19 + o sospechoso presenta un paro cardiorrespiratorio (PCR) y hay que iniciar maniobras de reanimación cardiopulmonar $(\mathrm{RCP})$ ? ¿Debemos reanimarlo considerando su pronóstico y la escasez de recursos? ¿Qué probabilidades tengo de contagiarme si participo de las maniobras? ¿Es "costo/efectivo" arriesgarme al contagio? ¿Debo realizar la RCP de acuerdo a los estándares International Liaison Committee On Reanimation (ILCOR) hasta aquí conocidos o debo considerar modificaciones en el algoritmo? ¿Qué consideraciones debo tener si el PCR se presenta mientras el paciente está en prono? Esta revisión busca responder en parte algunas de estas inquietudes, basándose en la literatura y recomendaciones que hasta ahora se han comunicado. Ciertamente, esta es escasa y está sujeta a sufrir progresivos y permanentes cambios, al menos por un tiempo, mientras logramos consolidar el conocimiento del comportamiento clínico global del COVID-19.

\section{Palabras clave:}

Paro cardiorrespiratorio, reanimación

cardiopulmonar, COVID-19, reanimación en prono

\section{Introducción}

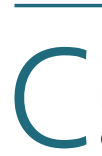

uando recién llevamos 60 años desarrollando la RCP e intentando resolver dudas que aún la evidencia no logra responder, la severa crisis sanitaria que ha golpeado al planeta en los últimos meses nos sitúa en un incómodo lugar, lleno de incertidumbres y de nuevas preguntas con difíciles respuestas o definitivamente, sin ellas.

Probablemente nunca hemos asistido a un escenario desconocido donde la evidencia se intenta buscar desesperadamente a partir de las experiencias recientemente compartidas por equipos de todas partes del mundo, o extrapoladas a partir de situaciones biológicas, epidemiológicas o clínicas similares previas. El conocimiento lo estamos generando sobre la marcha, presionados por el avance de la pandemia. Es por esta razón que se advierte que el contenido de este documento está vigente a la fecha de su elaboración y publicación, sin embargo, la posibilidad cierta de aparición de nuevos reportes o evidencias durante el desarrollo de esta pandemia, pueda cambiar lo aquí afirmado.

EI PCR es una instancia crítica por todos bien conocida, que inserta en el contexto del COVID-19, nos obliga a plantearnos inquietudes como las siguientes: 
cómo balancear el riesgo del reanimador y la comunidad versus las posibilidades de sobrevida y pronóstico del paciente, cómo balancear este pronóstico versus la escases de recursos físicos y humanos necesarios para dar atención a los pacientes críticos que esta pandemia amenaza con provocar; cómo adaptar las maniobras de reanimación clásicas que conocemos para hacerlas efectivas en contextos adversos, como, por ejemplo, el paciente en prono.

En este artículo revisaremos las recomendaciones y comunicaciones emanadas a partir de comités de expertos o de grupo de trabajo, que puedan sernos de utilidad para orientar las respuestas a estas preguntas y para definir conductas locales que cada uno deberá establecer de acuerdo con su realidad.

\section{Causa de PCR en COVID-19}

A la fecha, se reportan en el mundo 1.521.252 confirmados y 92.798 muertes. En América; 493.173 confirmados y 17.038 muertes[1].

En Chile contamos 6.501 confirmados con una letalidad de 0,99\%, según cifras del Ministerio de Salud (MINSAL). Ambos números aún muestran diariamente progresión ascendente.

Un reporte chino sobre 72.314 casos registrados hasta la mitad del mes de febrero recién pasado comunicaba las siguientes cifras: $2 \%$ al $4 \%$ de letalidad, $81 \%$ de los casos desarrolla síntomas leves, 14\% (12\% a 19\%, según otros reportes) presenta síntomas graves que motivan hospitalización y un 5\% (3\% a $6 \%$, según otros reportes) requiere ingreso a una Unidad de Pacientes Críticos[3]-[6].

Se ha visto que estos pacientes desarrollan fundamentalmente un síndrome de distress respiratorio agudo (SDRA) severo, con la consecuente hipoxemia profunda a la cual finalmente se le atribuye la causa final de muerte[7]. Sin embargo, también se ha reportado la aparición de complicaciones cardíacas mayores, como arritmias y falla cardíaca aguda. Los mecanismos de injuria miocárdica que se postulan son el aumento del estrés miocárdico a consecuencia de la falla respiratoria e hipoxemia, infección miocárdica directa por el virus y daño indirecto secundario a la respuesta inflamatoria sistémica. Se han encontrado elevación de biomarcadores, especialmente la troponina I de alta sensibilidad (hs-cTnl), en pacientes que presentan arritmias o falla cardíaca. Se asume que las arritmias se gatillan secundariamente a hipoxemia, desórdenes metabólicos, inflamación sistémica o miocarditis. También se ha visto que pacientes con elevación de troponina T (TnT) desarrollan con ma- yor frecuencia SDRA, arritmias ventriculares malignas e injuria renal aguda, además de presentar mayor mortalidad que aquellos con niveles de TnT normales. Inclusive, la miocarditis aguda y las arritmias ventriculares, pueden ser la primera manifestación del COVID-19, postulándose como la causa posible en casos de muerte domiciliaria. La isquemia coronaria aparece también como responsable de eventos críticos y estaría dado por aumento de la actividad protrombótica reflejada en el aumento de los niveles de dímero $D$ que se encuentra en estos enfermos. Es importante hacer notar que algunas opciones terapéuticas que se han sugerido como promisorias, como antimaláricos y antivirales, son potenciales tóxicos cardíacos, produciendo anormalidades en la conducción con prolongación del QT[8],[9]. También se ha reportado que entre los pacientes que mueren por COVID-19, un $11,8 \%$ tiene daño cardíaco mayor, con niveles elevados de troponina o presentación de un paro cardiorrespiratorio durante la hospitalización[10].

\section{Riesgo de reanimadores}

Como está establecido y ampliamente difundido, durante cualquier RCP, la primera prioridad debe ser la seguridad del personal que la realiza, declaración que no ha cambiado en el contexto de esta pandemia COVID-19. El International Liaison Committee on Resuscitation (ILCOR) en un intento por establecer cuál es el riesgo de los reanimadores al iniciar las maniobras de RCP, realizó una revisión de la evidencia existente al respecto, la cual a la fecha no se encuentra aún publicada, considerando estudios controlados y no controlados randomizados, estudios de cohortes, estudios controlados, reportes de casos y estudios en cadáveres[11]. No existen estudios que hayan explorado específicamente la transmisibilidad de COVID-19 durante las maniobras de RCP, por lo tanto, se considera aquellos que evalúan otros agentes. Existen además otros problemas, por una parte, no hay estudios que separen las diferentes maniobras que componen la reanimación como un todo y por otra, tampoco la evidencia es categórica para afirmar que alguna de éstas provoque diseminación del virus. No se separan las compresiones y la desfibrilación del manejo de la vía aérea y ventilación. El Resuscitation Council del Reino Unido (RCUK) menciona que existen guías internacionales que listan la RCP como un procedimiento generador de aerosoles, pero tampoco aclaran si esto es atribuible a la desfibrilación, compresiones torácicas o manejo de vía aérea y ventilación[12]. Sin embargo, considerar la posibilidad de que las compresio- 
nes torácicas provoquen aerosoles, parece plausible ya que, estas producen ventilación pasiva asociadas con la generación de volúmenes corrientes pequeños. Además, mientras se realiza esta maniobra, la persona permanece a muy corta distancia del paciente y de los demás reanimadores. La postura del RCUK es más categórica a pesar de la falta de evidencia y afirman que las compresiones torácicas sí constituyen un proceso generador de aerosoles y, por lo tanto, los reanimadores deben usar elementos de protección personal (EPP) adecuados para aquello, de acuerdo a la normativa local[12].

No existe evidencia para asegurar o descartar que la desfibrilación produzca aerosoles, pero en caso de que así ocurriera, la duración del proceso que provocaría la generación de éstos sería muy breve. Esto podría ser aún menos significativo, si se utilizan parches en lugar de las paletas para dar la descarga, lo cual evita el contacto directo del reanimador con el paciente.

En consideración a estas dificultades y sesgos, las recomendaciones de ILCOR atingentes a la RCP en contexto intrahospitalario o realizada por personal entrenado son las siguientes (se omiten aquellas que aluden a RCP extrahospitalaria por miembros de la comunidad):

- Sugiere que las compresiones torácicas y la RCP tienen el potencial de generar aerosoles (muy baja certeza/evidencia).

- Sugiere que durante la pandemia COVID-19, los profesionales de la salud deben usar elementos de protección personal (EPP) para procedimientos generadores de aerosoles, durante la reanimación (muy baja certeza/evidencia).

- Sugiere que podría ser razonable para los profesionales de la salud considerar desfibrilar antes de la postura de los EPP en aquellas situaciones en que el operador considere que el beneficio superará el riesgo (declaración de buenas prácticas).

\section{Recomendaciones para RCP en paciente COVID-19}

Ante este escenario y las implicancias mencionadas, resulta obvio que la RCP como la conocemos, se ve obligada a sufrir ciertas modificaciones conceptuales que parecen ser contrarias a lo que hemos preconizado majaderamente hasta aquí. Fundamentalmente en relación con dos puntos clave: la rapidez del inicio de las maniobras una vez hecho el diagnóstico y la no interrupción de las compresiones a todo evento, incluido el momento de la intubación orotraqueal, si es posible. Inclusive, las modificaciones atraviesan límites aún más incómodos, como evaluar la posibilidad de ventilación en forma adecuada, aún cuando la causa fuertemente sospechada sea la hipóxica.

A continuación, mencionaremos los principales aspectos de las recomendaciones emanadas desde el RCUK y de la AHA. No hay a la fecha una sola recomendación de consenso ILCOR. Tampoco existen guías locales[13],[14].

En primer lugar, es importante establecer que las recomendaciones se aplican para pacientes COVID-19 confirmados o sospechosos, de acuerdo a las definiciones vigentes[15].

Aun cuando se desconocen cifras exactas y el resultado del PCR y la RCP relacionados a COVID-19, se ha observado que la mortalidad de los pacientes críticos es alta y está relacionada a factores de riesgo bien identificados[8]-[10],[16]. Es relevante y recomendable en la medida de lo posible, considerar estos factores (edad avanzada, comorbilidad, marcadores de gravedad clínicos, de imágenes o de laboratorio al ingreso), y desarrollar en forma oportuna un plan preestablecido de toma de decisiones al enfrentar a un paciente COVID-19, el cual incluye conversación con paciente y familiares, de manera de enfrentar el evento crítico implementando acciones que logren alcanzar la mejor relación riesgo/beneficio considerando el pronóstico del paciente y el bienestar del personal y el resto de pacientes y comunidad, de acuerdo a la realidad de los recursos físicos y humanos disponibles. En el otro extremo, a la fecha, no existe evidencia que soporte el uso de ECMO para pacientes COVID-19.

Es así que en este especial escenario cobra especial relevancia el ser acucioso en la evaluación inicial del paciente, previendo la posibilidad de descompensación y evitando la necesidad de proceder a la intubación orotraqueal de urgencia, en una sala desprovista de las condiciones de seguridad indicadas (sala individual, presión negativa, idealmente).

Las recomendaciones consideran que el virus SARS-CoV-2 es altamente transmisible provocando alta morbimortalidad en la comunidad, con las graves consecuencias en los sistemas sanitarios que ya todos conocemos y a las cuales tememos. La transmisión se produce a través del contacto directo persona a persona vía gotitas y a través de la emisión de aerosoles.

Resulta obvio, y así se ha reconocido, que el personal de salud se encuentra dentro de los grupos de mayor riesgo de contraer la enfermedad[16]. Durante la RCP se agregan, además, tres factores que aumentan aún más el riesgo: la posibilidad cierta, aunque no haya sido evidenciada, de generación de gotitas y aerosoles durante las maniobras, la cercanía entre 
operadores y entre éstos y el paciente al momento de reanimar y la situación de estrés que atenta contra el orden y la rigurosidad para seguir protocolos de uso de EPP.

Por este motivo hay consenso en que todo personal de salud que se disponga a participar en una RCP debe usar irrenunciablemente EPP para precaución de gotitas y contacto al momento de monitorizar e intentar la desfibrilación. Para iniciar compresiones y manejo de ventilación y vía aérea, debe utilizar EPP para precaución de aerosoles, de acuerdo a las guías locales establecidas. Se recomienda tener kit de EPP preparados junto al carro de RCP.

Debe participar en la reanimación, el menor número de personas posible. Se sugiere el uso de dispositivos mecánicos para realizar las compresiones torácicas si se dispone de éste, para contribuir con este objetivo.

Para certificar el diagnóstico de PCR, en ningún caso chequear ventilación acercándose a cara del paciente, simplemente utilizar la búsqueda de pulso, capacidad de respuesta al estímulo verbal y, en el caso del paciente monitorizado, la pérdida de curvas de pletimosgrafía, de línea arterial o de capnografía si el paciente de encuentra intubado y se descarta la desconexión de circuitos o la extubación. Todo esto, independiente del ritmo que el trazado electrocardiográfico muestre.

Si hemos constatado la ocurrencia del PCR, debemos iniciar las maniobras sólo con compresiones y hacer diagnóstico de ritmo precozmente. Intentar desfibrilación lo antes posible, antes de iniciar compresiones si se diagnostica un ritmo desfibrilable. Esto aumenta la probabilidad de que haya retorno a circulación espontánea y de no requerir maniobras prolongadas.

La intervención de la vía aérea debe ser realizada por quien posea mayor experiencia y mejor desempeño, así mismo éste debe establecer la estrategia que mejor domine. La intubación orotraqueal es la opción y sólo debe ser realizada por expertos. Se debe instaurar lo antes posible interrumpiendo las compresiones cuando la maniobra se vaya a realizar. Inmediatamente después de instalado el tubo orotraqueal, se debe inflar el cuff y una vez confirmada la correcta instalación con la visualización de curva de capnografía en un monitor, es imperativo colocar un filtro HEPA (high-efficiency particulate air) entre el tubo y la bolsa ambú (antes de la toma de muestra para capnografía). Si hay disponible un circuito de ventilación mecánica, se debe instalar un segundo filtro en laram espiratoria. Sólo en este momento se puede dar la primera ventilación.
Si no se logra la intubación, la recomendación es utilizar un dispositivo supraglótico con filtro HEPA o realizar ventilación pasiva a través de una mascarilla de oxígeno común, cubierta con una mascarilla quirúrgica.

Se requiere ser en extremos riguroso con la manipulación de dispositivos como sondas de aspiración, mascarillas de oxígeno, máscaras faciales, hojas de laringoscopios y componentes de EPP. Todos deben ser depositados y eliminados estrictamente según lo dispuesto por los comités locales de infecciones. Se deben establecer protocolos locales para higienización de carro de paro y desfibrilador.

Para ventilar al paciente durante la reanimación la recomendación es usar $\mathrm{FIO}_{2}$ 1.0. Usando la modalidad presión control, ajustar hasta evidenciar elevación torácica (aproximadamente $6 \mathrm{ml} / \mathrm{K}$ de peso ideal). Ajustar FR a $10 \times$ minuto. Asegurar todas las conexiones y fijaciones del tubo orotraqueal para evitar desconexiones o extubación no previstas. Si el paciente retorna a circulación espontánea, se deben ajustar los parámetros del ventilador para alcanzar las metas de ventilación, oxigenación y hemodinámicas adecuadas.

Las recomendaciones de RCP en el caso de la paciente obstétrica no difieren de las mencionadas. Es importante que los equipos de obstetras y neonatólogos inicien inmediatamente la preparación y colocación de EPP para estar listos a tiempo para realizar la cesárea peri mortem, la cual no debe tardar más de cuatro minutos.

La Figura 1 muestra un resumen de los puntos más relevantes de la RCP que se modifican en este contexto y la Figura 2, cómo se identifican en el algoritmo que propone la American Heart Association.

\section{Reanimación en prono}

La efectividad de la RCP en prono es desconocida, aunque hay algunos estudios con pequeño número de pacientes y reportes de casos que comunican resultados exitosos y el logro de altas presiones arteriales medias. Si existe riesgo mayor de desconexión o extubación al dar vuelta al paciente, se recomienda iniciar las maniobras en esa misma posición para evitar mayor riego de aerosolización. Para desfibrilar, se pueden instalar parches en posición anteroposterior o usar paletas o parches posterolaterales (uno en la línea axilar izquierda y el otro sobre la escápula derecha). También se pueden situar bilateralmente sobre ambas líneas axilares medias. Para realizar las compresiones las manos se apoyan sobre la línea media como es habitual, entre T7 y T10. Si no se logra el retorno 


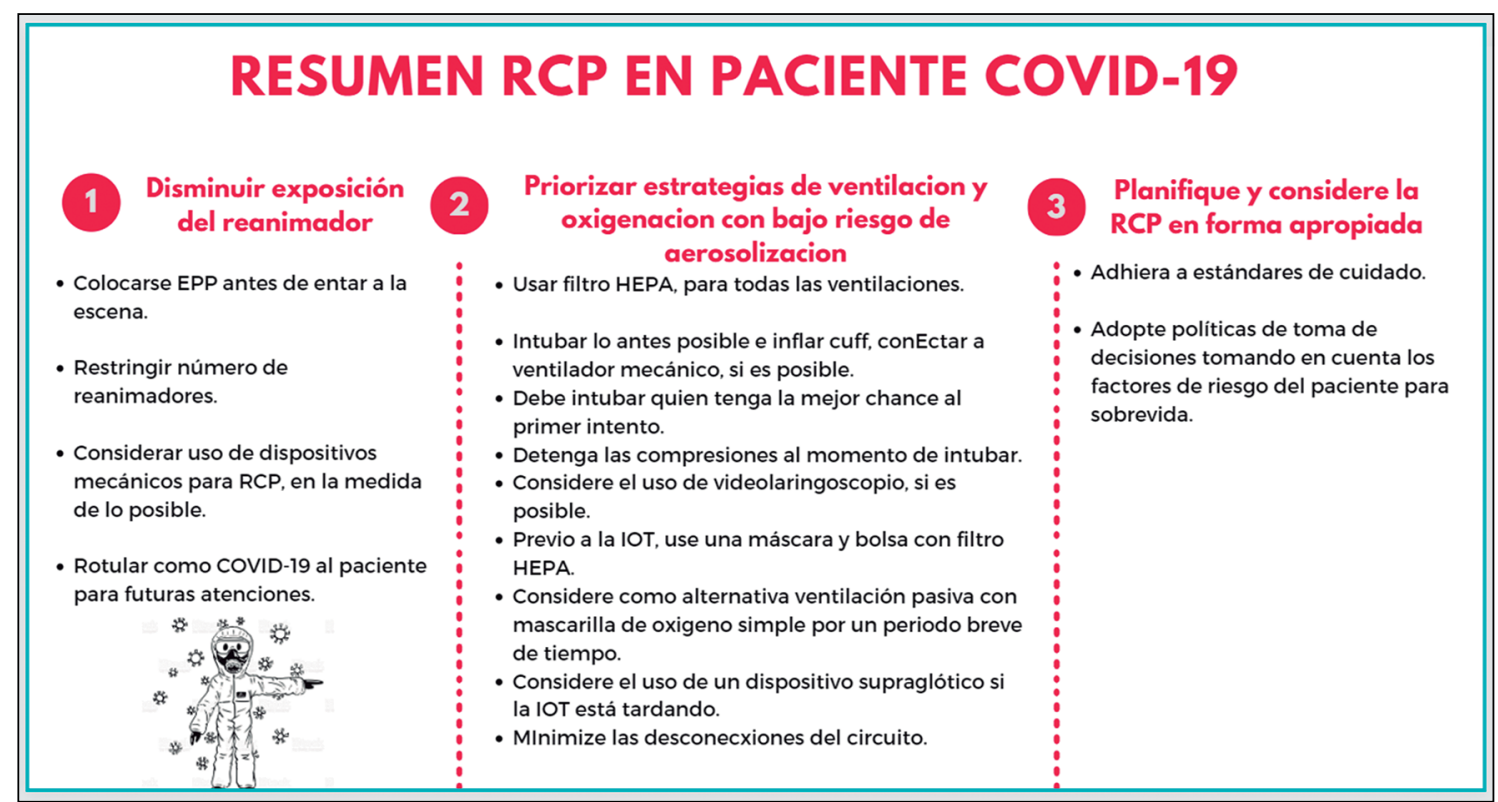

Figura 1. Resumen de modificaciones a tener en cuenta en las recomendaciones para RCP en pacientes COVID-19, tomada de Resuscitation Council UK.

a la circulación espontánea durante la reanimación en prono, el equipo deberá tomar la decisión de dar vuelta al paciente buscando mayor probabilidad de éxito, sopesando el riesgo, de acuerdo a cada situación particular[17].

\section{¿Podemos plantearnos no reanimar a los pacientes COVID-19?}

Es claro que la pandemia actual nos ha quitado las certezas con las que enfrentábamos el PCR y la RCP, forzándonos a renunciar a declaraciones a las que históricamente hemos adherido como "no hay daño en tratar", "hacer todo lo que se pueda". O a ampliar el significado de otras como "no hacer daño". Como señalan Fritz y Perkins, al iniciar maniobras de RCP ya no sólo debemos pensar en el daño individual al paciente, sino también en el que atañe al personal de la salud y el resto de la comunidad[18].

Es discutido, con escasa base de evidencia, si las diferentes partes que componen la RCP provocan o no aerosoles. La probabilidad de éxito de las maniobras, dependen de la oportunidad con que se instauren y de la causa, así como del estado general previo del paciente. La seguridad de los reanimadores es intransable y, por lo tanto, la colocación correcta y completa de EPP no debe omitirse bajo ningún punto de vista.
Si bien la principal causa que generaría el PCR en estos pacientes, pudiera ser la hipóxica, no es la única y no se puede asumir como tal. El pronóstico de sobrevida en PCR en pacientes COVID-19 es desconocido. Por lo tanto, parece inadecuado asumir a todo evento que la causa es no tratable, como pudiera ser una falla respiratoria catastrófica, que el paciente es irrecuperable y, por lo tanto, establecer a priori que no se iniciarán maniobras si el evento sucede.

Es extremadamente necesario realizar un análisis profundo de la situación clínica de cada paciente ingresado a una unidad de emergencia o de paciente crítico. Sostener una conversación con el paciente y/o la familia, en lo posible, donde se transparenten las dificultades y desafíos que plantea un eventual $P C R$, tanto desde el punto de vista del paciente, como de las consecuencias que trae el riesgo de contagio del personal de salud para ellos y del resto de la comunidad. Siempre considerar la situación local de recursos físicos y humanos. El enfoque fundamental debe estar dirigido hacia la prevención de la ocurrencia del PCR y la generación de toda medida que sea necesaria para que éste finalmente no ocurra. Las intervenciones y sus límites deben ser planteados de acuerdo con las realidades locales, teniendo en mente que la decisión de no iniciar maniobras de reanimación es una de las más difíciles que un clínico puede enfrentar[19]. 


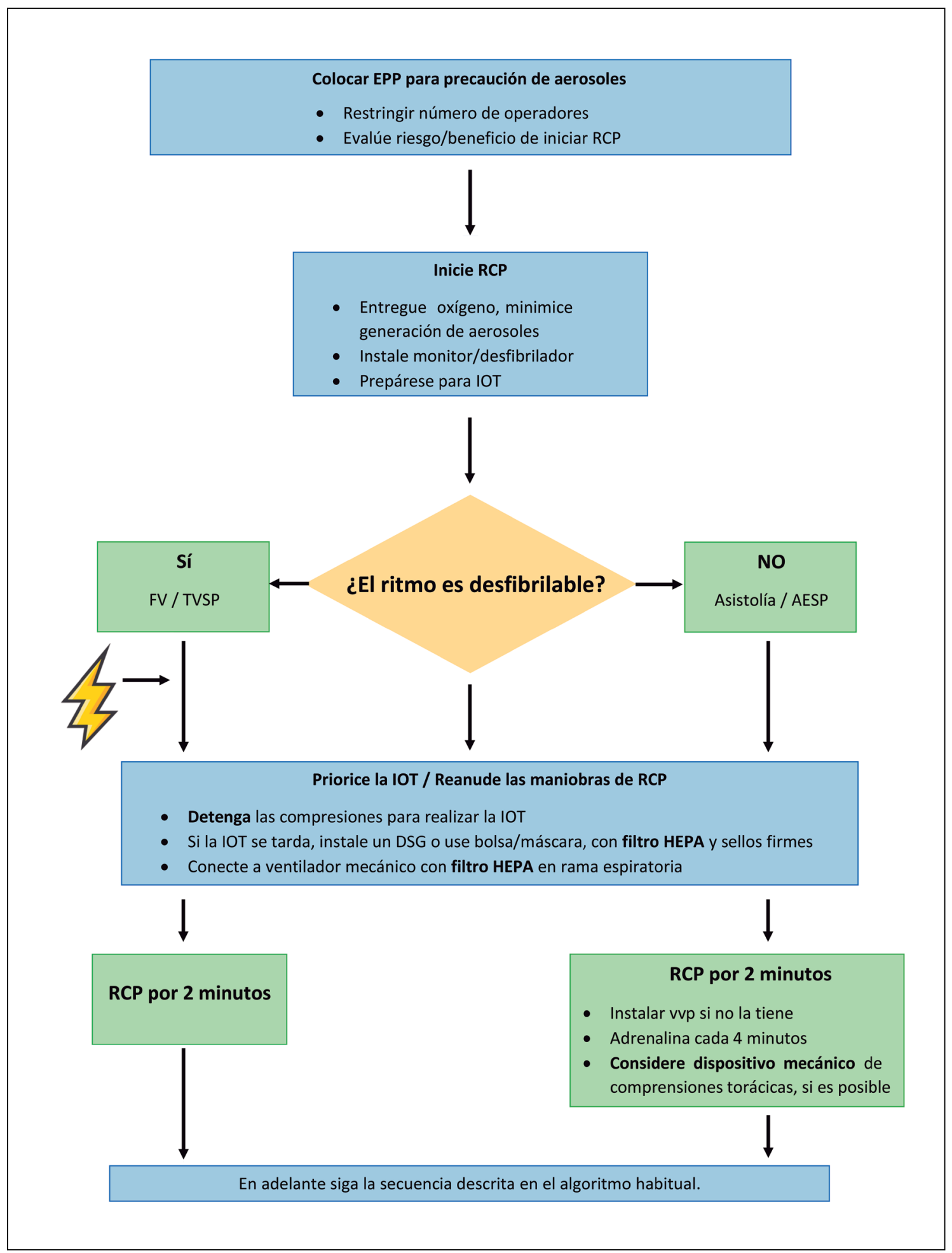

Figura 2. Algoritmo de RCP avanzada para paciente adulto COVID-19 propuesto por la American Heart Association, modificado. 


\section{Referencias}

1. https://www.who.int/docs/ default-source/coronaviruse/ situation-reports/20200410sitrep-81-covid-19. pdf?sfvrsn=ca96eb84_2

2. https://cdn.digital.gob.cl/public files/Campa\%C3\%B1as/CoronaVirus/Reportes/10.04.2020_ Reporte_Covid19.pdf

3. 10. Novel Coronavirus Pneumonia Emergency Response Epidemiology Team. 2020. The epidemiological characteristics of an outbreak of 2019 novel coronavirus diseases (COVID-19) in China. Zhonghua Liu Xing Bing Xue Za Zhi 41:145151. doi:10.3760/cma.j.is sn.0254-6450.2020.02.003.

4. Bialek S, Boundy E, Bowen V, Chow N, Cohn A, Dowling N, et al.; CDC COVID-19 Response Team. Severe Outcomes Among Patients with Coronavirus Disease 2019 (COVID-19) - United States, February 12-March 16, 2020. MMWR Morb Mortal Wkly Rep. 2020 Mar;69(12):343-6. https://doi. org/10.15585/mmwr.mm6912e2 PMID:32214079

5. Wu Z, McGoogan JM.

Characteristics of and Important Lessons From the Coronavirus Disease 2019 (COVID-19) Outbreak in China: Summary of a Report of 72314 Cases From the Chinese Center for Disease Control and Prevention. JAMA. 2020 Feb;323(13):1239-42. https://doi.org/10.1001/ jama.2020.2648 PMID:32091533

6. Guan WJ, Ni ZY, Hu Y, Liang WH, Ou CQ, He JX, et al.; China Medical Treatment Expert Group for Covid-19. Clinical Characteristics of Coronavirus Disease 2019 in China. N Engl J Med. 2020 Feb;NEJMoa2002032. https://doi.org/10.1056/

NEJMoa2002032; epub ahead of print. PMID:32109013

7. Huang $X$, Wei F, Hu L, Wen L, Chen K. Epidemiology and Clinical Characteristics of COVID-19. Arch Iran Med. 2020 Apr;23(4):268-71. https://doi. org/10.34172/aim.2020.09 PMID:32271601

8. Kochi AN1. Tagliari AP2, Forleo GB3, Fassini GM1, Tondo C1,4. Cardiac and arrhythmic complications in Covid-19 patients [Epub ahead of print]. J Cardiovasc Electrophysiol. 2020 Apr;•••: https://doi.org/10.1111/ jce. 14479.

9. Akhmerov A, Marban E. COVID-19 and the Heart. Circulation Research [Internet]. Ovid Technologies (Wolters Kluwer Health); 2020 Apr 7; https://doi.org/10.1161/ CIRCRESAHA.120.317055.

10. Zheng YY, Ma YT, Zhang JY, Xie X. COVID-19 and the cardiovascular system. Nat Rev Cardiol. 2020 Mar 5th, online first publication. DOI: 10.1038/ s41569-020-0360-5.

11. Couper K, Taylor-Phillips S, Grove A, Freeman K, Osokogu O, Court R, et al. Perkins GD on behalf of the International Liaison Committee on Resuscitation. COVID-19 Infection risk to rescuers from patients in cardiac arrest. Consensus on Science with Treatment Recommendations [Internet] Brussels, Belgium: International Liaison Committee on Resuscitation (ILCOR), 2020 March 30. Available from: http:// ilcor.orghttps://costr.ilcor.org/ document/covid-19-infectionrisk-to-rescuers-from-patients-incardiac-arrest? status=draftFinal

12. https://www.resus.org.uk/ media/statements/resuscitationcouncil-uk-statementson-covid-19-coronavirus- cpr-and-resuscitation/

faqs-on-cpr-during-the-covid-19pandemic/

13. https://www.resus.org.uk/ media/statements/resuscitationcouncil-uk-statements-oncovid-19-coronavirus-cpr-andresuscitation/covid-healthcare/

14. Dana P. Edelson, Comilla Sasson, Paul S. Chan, Dianne L. Atkins, Khalid Aziz, Lance B. Becker, Robert A. Berg, Steven M. Bradley, Steven C. Brooks, Adam Cheng, Marilyn Escobedo, Gustavo E. Flores, Saket Girotra, Antony Hsu, Beena D. KamathRayne, Henry C. Lee, Rebecca E. Lehotzky, Mary E. Mancini, Raina M. Merchant, Vinay M. Nadkarni, Ashish R. Panchal, Mary Ann R. Peberdy, Tia T. Raymond, Brian Walsh, David S. Wang, Carolyn M. Zelop, Alexis Topjian. Interim Guidance for Basic and Advanced Life Support in Adults, Children, and Neonates With Suspected or Confirmed COVID-19:From the Emergency Cardiovascular Care Committee and Get With the Guidelines $₫$-Resuscitation Adult and Pediatric Task Forces of the American Heart Association in Collaboration with the American Academy of Pediatrics, American Association for Respiratory Care, American College of Emergency Physicians, The Society of Critical Care Anesthesiologists, and American Society of Anesthesiologists: Supporting Organizations: American Association of Critical Care Nurses and National EMS Physicians. Circulation. 2020;(April): https://doi.org/10.1161/ circulationaha.120.047463.

15. Ministerio de Salud, División Planificación Sanitaria. Departamento Epidemiología. Ordinario N 895, 18 de Marzo 2020. Informa definición vigente 
para fase 4.

16. Thomas-Rüddel D, Winning J, Dickmann $P$, Ouart D, Kortgen A, Janssens U, et al. Coronavirus disease 2019 (COVID-19): update for anesthesiologists and intensivists March 2020. Anaesthesist. 2020 Mar; https:// doi.org/10.1007/s00101-02000760-3 PMID:32211920

17. Working Group of the Resuscitation Council (UK), Neuroanaesthesia Society of
Great Britain and Ireland and Society of British Neurological Surgeons. Management of cardiac arrest during neurosurgery in adults. Guidelines for healthcare providers. Date of first publication: August2014, Date of Review July 2019. https:// www.resus.org.uk/publications/ management-of-cardiac-arrestduring-neurosurgery-in-adults/ 18. Fritz Z., Perkins G.
Cardiopulmonary resuscitaction after hospital admission with COVID-19. BMJ 2020;369:m1387 doi: 10.1136/ bmj.m1387 (Published 6 April 2020)

19. Mahase E., Kmietowicz Z. COVID-19: Doctors are told not to perform CPR on patients in cardiac arrest. BMJ 2020;368:m1282 doi: 10.1136/ bmj.m1282 (Published 29 March 2020) 